AperTO - Archivio Istituzionale Open Access dell'Università di Torino

\title{
Resistance to Anti-EGFR Therapy in Colorectal Cancer: From Heterogeneity to Convergent
} Evolution.

\section{This is the author's manuscript}

Original Citation:

\section{Availability:}

This version is available http://hdl.handle.net/2318/154348

since 2016-06-14T12:38:23Z

Published version:

DOI:10.1158/2159-8290.CD-14-0462

Terms of use:

Open Access

Anyone can freely access the full text of works made available as "Open Access". Works made available under a Creative Commons license can be used according to the terms and conditions of said license. Use of all other works requires consent of the right holder (author or publisher) if not exempted from copyright protection by the applicable law. 


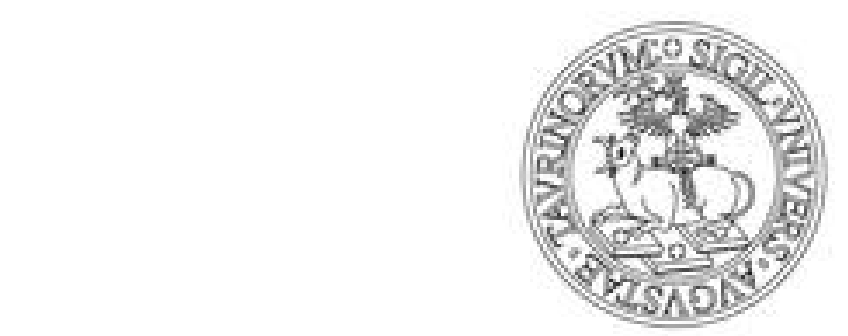

\section{UNIVERSITÀ DEGLI STUDI DI TORINO}

This is an author version of the contribution published on:

Misale S,Di Nicolantonio F,Sartore-Bianchi A,Siena S,Bardelli A Resistance to Anti-EGFR Therapy in Colorectal Cancer: From Heterogeneity to Convergent Evolution.

CANCER DISCOVERY (2014) 4

DOI: $10.1158 / 2159-8290 . C D-14-0462$

The definitive version is available at:

http://cancerdiscovery.aacrjournals.org/cgi/doi/10.1158/2159-8290.CD-14-04 62 


\title{
Resistance to anti-EGFR therapy in colorectal cancer: from heterogeneity to convergent evolution
}

\author{
Sandra Misale ${ }^{1,2}$, Federica Di Nicolantonio ${ }^{1,2}$, Andrea Sartore-Bianchi ${ }^{3}$, Salvatore \\ Siena $^{3}$ and Alberto Bardelli ${ }^{1,2,4,}$ \\ 1 \\ University of Torino, Department of Oncology, SP 142, Km 3.95, 10060 Candiolo, Torino, \\ Italy ${ }^{2}$ Candiolo Cancer Institute - FPO, IRCCS, Candiolo, Torino, Italy ${ }^{3}$ Department of Hematology \\ and Oncology, Niguarda Cancer Center, Ospedale Niguarda Ca' Granda, Milan, Italy ${ }^{4}$ FIRC \\ Institute of Molecular Oncology (IFOM), Milano, Italy
}

Running title: Resistance to anti-EGFR therapy in colorectal cancer

Keywords: Colorectal cancer, EGFR targeted therapy, cetuximab, panitumumab, primary resistance, acquired resistance, KRAS, BRAF, NRAS, MET

$\S$ Correspondence to: Alberto Bardelli, University of Torino, Department of Oncology, SP $142 \mathrm{Km}$ 3.95, 10060 Candiolo, Torino, Italy. Phone: +39 011 9933235; Fax: +39 0119933225 ; E-mail: alberto.bardelli@unito.it

Disclosure of Potential Conflicts of Interest: The authors disclose no potential conflicts of interest.

Word Count: 4721 words (excl. references)

Number of References: 90

Figures and tables: 4 figures and 1 table 


\begin{abstract}
The EGFR-targeted antibodies cetuximab and panitumumab are used to treat metastatic colorectal cancers. Mutations in KRAS, NRAS and BRAF and amplification of ERBB2 and MET, drive primary (de novo) resistance to anti-EGFR treatment. Recently, the emergence of alterations in the same genes was detected in patients who responded to EGFR blockade and then relapsed. These results enlighten a striking overlap between genes that, when mutated, drive primary and secondary resistance to anti-EGFR antibodies. Remarkably, while the mechanisms of resistance are genetically heterogeneous, they biochemically converge on key signaling pathways. This knowledge is being translated in the rational design of additional lines of therapy.
\end{abstract}

Statement of Significance: Anti-EGFR targeted therapies are used for the treatment of metastatic colorectal cancer. Molecular heterogeneity impairs their efficacy by fuelling de-novo and acquired resistance. In this review, we highlight how genetically distinct resistance mechanisms, biochemically converge on a limited number of signalling pathways which can be therapeutically intercepted. 


\section{The heterogeneous molecular landscape of colorectal cancer}

On December 27 1831, Charles Darwin leaves Plymouth Harbor on board of the H.M.S. Beagle to begin a long journey that, more than any other transformed scientific knowledge. While traveling, Darwin had the possibility to observe and collect samples from heterogeneous animal and vegetal species around the landscape of multiple continents. His rigorous scientific method established the bases of the unifying theory of life sciences, ultimately explaining the diversity of life.

Planet Earth hosts ecosystems endowed with extraordinarily diverse environmental conditions; the latter exert selective pressures, which enable evolution. Comparable selective pressures foster the development of parallel evolutionary results of unrelated species living in distinct ecosystems (convergent evolution). Classic examples of this phenomenon are the convergent evolution of wings or fins in birds and mammals.

In addition to his exceptional intuitive thinking, Darwin was able to "quantify" his observations by visiting several locations and analyzing the results of evolutionary processes in all of these environments.

In 1976, Peter Nowell stated that "... tumor progression results from acquired genetic variability within the original clone allowing sequential selection of more aggressive sub-lines" and, most importantly, that "more research should be directed toward understanding and controlling the evolutionary process in tumors before it reaches the late stage usually seen in clinical cancer" (1).

Each individual tumor can be seen as a microcosm under incessant variation based on genetic diversity (heterogeneity), selection and evolution; the very same mainstays on which life is based, although tumors proceed through them at a much faster pace. Just like Darwin did more than a century ago with the complexity of speciation, research in oncology strives to understand cancer's intricacy.

The ability to explore (which we define as the ability to molecularly annotate) cancer genomes can be seen as a modern version of the H.M.S. Beagle. By applying next-generation sequencing (NGS) technologies to scan the cancer genome, the oncology community has recently (re)discovered molecular heterogeneity in tumor samples (2), including those of colorectal origin (3).

Colorectal cancer (CRC), the third most common cancer type in western countries, affects more than 200,000 patients worldwide every year (4). Screening, surgery and medical therapies are successful in the management of early-stage CRC, but far less efficacious in advanced stages of the disease. A key reason of the limited success of CRC-directed therapies is its intrinsic heterogeneity, which is more prominent in the metastatic setting $(5,6)$. Molecular characterization of CRCs revealed that heterogeneity plays an important role especially in the context of resistance to therapy. Over half CRCs display heterogeneous genetic alterations in genes involved in EGFR 
signaling, which negatively impact response to the monoclonal antibodies cetuximab and panitumumab. Molecular heterogeneity has been recognized as pivotal in the evolution of clonal populations during anti-EGFR therapies. In this review, we provide an outline of how genetic diversity (molecular heterogeneity) influences primary (de novo) and secondary (acquired) resistance to EGFR targeted therapies in CRC.

\section{Mechanisms of primary resistance to EGFR targeted therapy in CRC}

\section{Known culprits}

The EGFR-directed monoclonal antibodies cetuximab and panitumumab were approved to treat chemorefractory metastatic colorectal cancer (mCRC) patients in 2004 and 2006, respectively (Fig. 1). Both drugs have very similar efficacy, achieving objective response rates of approximately $10 \%$ when used as monotherapy for irinotecan- and/or oxaliplatin- refractory $\mathrm{mCRC}(7,8)$. Investigations on the molecular basis of response to EGFR blocking antibodies started in 2005 and were based on retrospective analyses of archived tumor tissue from subsets of patients participating in clinical trials (9). Since then, a rapidly accumulating body of knowledge has indicated that resistance to EGFR blockade in $\mathrm{mCRC}$ is related to constitutive activation of signaling pathways downstream of EGFR. Mutations in KRAS occurring at codons 12 and 13 were the first to be causally implicated in resistance to EGFR targeted monoclonal antibodies initially in small patient cohorts $(10,11)$. Randomized phase III studies provided compelling evidence that led regulatory authorities to exclude chemorefractory $\mathrm{mCRC}$ patients with tumors bearing KRAS mutations from treatment with single-agent cetuximab or panitumumab $(12,13)$. In 2009, the analysis of KRAS codon 12 and 13 mutations as a test to restrict the use of cetuximab in combination with chemotherapy to first-line $\mathrm{mCRC}$ patients with wild-type tumors gained regulatory approval $(14,15)$.

Since not all KRAS wild type patients benefit from treatment with EGFR directed therapy, research has flourished to identify additional biomarkers of resistance that could account for the heterogeneity in clinical response. Sequencing studies revealed that while over $80 \%$ of $K R A S$ variants occur in exon 2 at codons 12 and 13, oncogenic mutations also affect KRAS codons 59, 61,117 and 146 (16-18). Additional mutations of the NRAS isoform occur at codons 12, 13 and 61 in approximately $3-5 \%$ of CRC samples (19). Figure 2 summarizes the incidence of $R A S$ mutations in exon 2 (including codons 12 and 13), exon 3 (comprising codons 59 and 61) and exon 4 (which includes codons 117 and 146). Mutations in KRAS or NRAS lead to continuous activation of downstream ERK signaling regardless of whether the EGFR is pharmacologically inactivated. While the role of the canonical exon 2 mutations is considered uncontroversial, the exact properties of the less frequent mutations have not been fully elucidated. However, data from retrospective studies indicate that RAS mutations occurring beyond KRAS exon 2 could also underlie lack of 
response to single-agent cetuximab or panitumumab in chemorefractory mCRC patients (20-23). Multiple studies have recently shown that mutations in KRAS exons 3 and 4 , or NRAS exons 2 to 4 can also predict lack of clinical benefit to EGFR targeted antibodies given in combination with firstline chemotherapy (24-26).

While the presence of RAS mutations accounts for around $50-60 \% \mathrm{mCRC}$ patients refractory to EGFR blockade, molecular alterations in additional nodes of the EGFR signaling network also appear to be clinically relevant. Among them, $B R A F$ mutations occur in approximately $5-8 \%$ of the cases and are associated with poor prognosis in the metastatic setting. Experiments in CRC cells and mouse models demonstrated a strong causal relationship between the presence of $B R A F$ V600E and resistance to cetuximab or panitumumab (27-29). Several reports have shown a significant negative predictive value for $B R A F \mathrm{~V} 600 \mathrm{E}$ mutations in relation to response to singleagent cetuximab or panitumumab $(20,21,23,27,30,31)$. Recent studies in patients receiving EGFR targeted monoclonal antibodies in combination with first-line chemotherapy have not found a statistically significant correlation between $B R A F \mathrm{~V} 600 \mathrm{E}$ mutations and response (presumably due to lack of statistical power), but they confirmed the link between BRAF V600E mutations and poor prognosis in $\mathrm{mCRC}(24,26)$.

\section{Under scrutiny}

When combined, RAS and BRAF mutations account for over $60 \% \mathrm{mCRC}$ patients who show denovo resistance to EGFR targeted monoclonal antibodies. Beyond RAS and BRAF point mutations, numerous genetic alterations in genes implicated in EGFR signaling play a role in de novo resistance. Importantly, although molecularly heterogeneous, these alterations biochemically converge on activation of the RAS-MEK-ERK pathway.

KRAS gene amplification occurs in $1-2 \%$ of $C R C$ cases and has been reported to be nearly always mutually exclusive with KRAS mutations $(18,32,33)$. KRAS gene amplification has been shown to cause resistance to cetuximab in functional genetics experiments and has been associated with lack of response to anti-EGFR treatment $(32,33)$. Given the low prevalence of KRAS gene amplification, its association with refractoriness to EGFR blockade did not reach statistical significance. Analysis from the TCGA CRC database (34) has revealed that gene amplification can also occur in NRAS, BRAF and $C R A F$, at a very low prevalence ( $<1 \%$ cases for individual genes), and the clinical relevance of these findings is unknown.

Additional genetic mechanisms have been proposed to activate the EGFR-RAS pathway in the absence of molecular alterations affecting RAS or its immediate downstream effectors. Genetic aberrations of the receptor tyrosine kinases ERBB2 and MET have been shown to bypass EGFR signaling and activate the MEK-ERK cascade. ERBB2 gene amplification was found in a small fraction of $R A S$ and $B R A F$ wild-type $\mathrm{mCRC}$ patient-derived xenografts that were insensitive to 
cetuximab treatment. These results were corroborated by the identification of ERBB2 amplification in samples from $\mathrm{mCRC}$ patients who did not benefit from EGFR targeted treatment (29). Concordant data was obtained by Yonesaka and colleagues who showed that activation of ERRB2 signaling dependent either by gene amplification or overproduction of the ERBB3 ligand, heregulin was present in a subset of $\mathrm{mCRC}$ patients exhibiting de-novo resistance to cetuximab-based therapy (35). Another tyrosine kinase receptor, $M E T$, is amplified in a small fraction (2\%) of $\mathrm{mCRC}$ samples unselected for their sensitivity to anti-EGFR therapy $(34,36-38)$. Once again, amplified $M E T$ was found in a small fraction of $R A S$ and $B R A F$ wild-type $\mathrm{mCRC}$ patient derived xenografts that were insensitive to cetuximab treatment (38). Therefore these pathways may offer primary 'escape mechanisms', allowing tumors to circumvent one pathway that has been pharmacologically blocked.

\section{By-standers or partners in crime?}

The overall scenario is further complicated by the existence of additional CRC genetic alterations in EGFR signaling which might confer resistance to cetuximab or panitumumab. For example, the PI3K-AKT-PTEN pathway can also be triggered by EGFR activation; therefore, several studies were conducted to define whether molecular alterations of these genes could also impair response to EGFR targeted monoclonal antibodies. Results obtained by multiple laboratories associate PIK3CA exon 20 mutations with unresponsiveness to anti-EGFR monoclonal antibodies; however the correlation is not strong enough to be applied as a clinically valuable negative predictive marker of response, possibly due the relatively small sample size of each study and the confounding effect of concomitant chemotherapy administration (20,39-48). PTEN status is also associated with a lack of response, but also in this case, results remain inconclusive partially because of difficulties in assessing the status of PTEN in clinical specimens $(30,40,42,49-56)$. Moreover, PIK3CA and PTEN alterations (around $10-15 \%$ overall) often co-occur with KRAS or BRAF mutations $(20,30,34,44)$, a feature, which further complicates their assessment. In summary, the role of PIK3CA and PTEN in conferring resistance to EGFR directed therapy in CRC remains highly controversial.

\section{Other suspects}

The genetic mechanisms described above do not account for the totality of patients who show clinical resistance to anti-EGFR drugs. Indeed, for approximately $10 \%$ of cases the genetic alteration which confers de-novo resistance is presently unknown. We hypothesize that when a patient fails to respond to EGFR treatment the most likely cause is the occurrence of a yet to be reported genetic alteration in either a RTK, or a downstream amplifier of the RTK initiated signal or a key node of the EGFR signaling pathway. 
Most likely these will be found in genetic alterations in known oncogenes, such as amplification or translocations of RTKs identified by the TCGA in CRC samples that do not harbor RAS or BRAF mutations such as NTRK, RET, ALK or ROS1 (34). These additional oncogenic events are present at low prevalence (1-5\%) and analyses of large datasets will be required for their clinical validation.

Alternatively, it is possible that well known alleles (such as $R A S$ mutations) are present in the tumor at prevalence that cannot be detected by commonly used techniques. The low sensitivity issue has its roots in tumor heterogeneity. Tissue biopsies represent a small fraction of the entire tumor burden. This assumption means that, because of intra-tumor and/or inter-metastases heterogeneity, analysis of tissue from an individual biopsy may not capture its entire molecular complexity. The analysis of multiple biopsies form a single patient, revealed the presence of several sub-clones which can be present or absent in different metastases or primary site. Furthermore, the same single lesion can harbor more than one independent clone $(2,57,58)$. These observations are particularly relevant when considering that previous studies mainly involved analysis of KRAS exon 2 mutations and that the most commonly used techniques (Sanger sequencing) has a limit of detection of approximately 15-20\% (59). Of interest, it has been shown that more sensitive approaches such as pyrosequencing or digital PCR can increase the detection of mutant RAS alleles which in turns could translate into the detection of additional refractory patients $(22,57,58,60,61)$.

Finally, while in this report we focused mainly on genetic heterogeneity as a basis for the complexity observed in resistance to EGFR inhibition in CRC, non-genetic mechanisms could also play a role in resistance to EGFR blockade (and are definitely relevant with other targeted agents in different cancers). Intriguingly, in biopsies from patients who relapsed upon cetuximab or panitumumab therapy only a fraction of cells carry RAS mutations, suggesting that wild-type cells can also survive the treatment (62). This suggests that non-genetic mechanisms could also play a role in driving acquired resistance to EGFR blockade. For example, a recent report shows that sensitive (wild type) cells can survive in the presence of cetuximab when in the company of their resistant derivatives. Notably, it was found that cells bearing acquired RAS mutations over-secrete the EGFR ligands TGFa and amphiregulin, which protect the surrounding wild-type cells (63). This paracrine network could potentially be targeted to increase the efficacy of anti-EGFR therapies.

\section{What drives sensitivity to EGFR blockade in CRC? : The EGFR ligands hypothesis}

The molecular basis underlying response to EGFR targeted therapies in CRC remains obscure. Several studies showed that increased EGFR gene copy number correlates with response to cetuximab or panitumumab, in preclinical models and in retrospective clinical analyses $(9,64-69)$. 
Nevertheless this alteration is not currently used as a predictive biomarker because of the difficulties in inter-laboratory reproducibility of the diagnostic assay(70).

Although the molecular bases of sensitivity to EGFR blockade are unclear, the clinical efficacy of EGFR targeted monoclonal antibodies provides evidence that EGFR signaling plays a prominent role in certain CRCs. We propose that dependency on EGFR ligands (via a paracrine-juxtacrine network) is the main oncogenic driver in the CRC that display sensitivity to cetuximab and panitumumab. In these CRCs, activation of the EGFR RAS-MEK axis is not sustained by mutations of downstream effectors rather may be achieved by overproduction of EGFR ligands. Classical studies on viral oncogenes led to the identification of EGFR ligands as being equally effective in triggering cell transformation as RAS. In these colorectal tumors anti EGFR antibodies may act by interfering with ligand-dependent activation of EGFR leading to downregulation of the receptor from the cell surface $(71,72)$.

\section{Mechanisms of secondary resistance to anti-EGFR therapy in CRC}

\section{Mutations of the EGFR extracellular domain}

In a subset of CRC patients, addition of anti-EGFR monoclonal antibodies to the conventional chemotherapeutic regimens expands response rates, increases progression-free survival, and improves the quality of life. However, the duration of this response is only transient and does not last more than 3-12 months, after which secondary resistance occurs. Several studies based on preclinical models and tumor samples obtained at relapse identified molecular mechanisms, which lead to acquired resistance to EGFR blockade in CRC.

Montagut and colleagues discovered a point mutation in the extracellular domain of EGFR (S492R) in a CRC cell line made resistant to cetuximab (73). This mutation impairs binding of the antibody to the receptor and was found also in very few patients at relapse after cetuximab treatment. The S492R mutation does not interfere with binding of panitumumab. Thus, patients with tumors showing the S492R mutation at relapse could be, in principle, treated with panitumumab. Indeed, they reported that a patient harboring the S492R allele as mechanism of secondary resistance to cetuximab was subsequently treated with panitumumab and responded transiently to this therapy. Notably the crystal structure of cetuximab bound to the extracellular domain of the EGFR indicates that S492R likely interfere with ligand binding (74). Since other residues in the extracellular region could equally affect the binding of cetuximab to the EGFR we postulated that molecular profiling of these regions in tumors which developed resistance to EGFR antibodies may reveal additional mutations capable of conferring acquired resistance to cetuximab or panitumumab. 


\section{Amplification of receptor tyrosine kinases}

Amplification of genes encoding for receptor tyrosine kinases is also associated with secondary resistance to anti-EGFR monoclonal antibodies. ERBB2 or MET gene amplifications were described as drivers of acquired resistance to EGFR blockade in cell models and patients samples $(35,38)$. Several reports confirmed the initial results on the emergence of METgene amplification in patients who develop acquired resistance to EGFR blockade $(75,76)$.

\section{Mutations in RAS genes}

The most common molecular mechanisms that drive secondary resistance to anti-EGFR therapy in $\mathrm{CRC}$ are genetic alterations of the KRAS gene (both point mutations and gene amplification). The emergence of $N R A S$ and $B R A F$ mutations is likewise associated to secondary resistance $(62,77$ 79).

Of note, KRAS, NRAS and BRAF mutations as well as amplification of the MET or ERBB2 genes are also key drivers of primary resistance to anti EGFR antibodies in CRC. Remarkably, while the genetic drivers of primary resistance are usually homogeneously within an individual tumor, more than one driver alteration can emerge in a single tumor at relapse.

CRC cell lines made resistant to cetuximab or panitumumab, showed the concomitant presence of diverse genetic mechanisms: for instance, in one single resistant cell model we were able to identify multiple KRAS mutations together with NRAS mutant clones as well (78). The genetic landscapes of cell models are generally considered as molecularly homogeneous, however these experiments suggest that the resistant population may arise upon selection of multiple clones which were presumably already present at the beginning of treatment.

The intrinsic genetic heterogeneity, which sustains acquired resistance to anti EGFR antibodies in preclinical models, was confirmed in clinical samples from CRC patients at relapse after anti-EGFR treatment. Bettegowda and colleagues analyzed circulating cell free tumor DNA obtained from plasma samples of CRC patients at relapse with ultra-sensitive technologies (79). Seventy-six genetic alterations were detected at resistance which were all absent in samples from the same patients at the beginning of treatment. Half of the alterations were in KRAS codons 12 or 13; mutations in $B R A F(\mathrm{~V} 600 \mathrm{E})$ were observed in two patients. Interestingly, in two patients mutations in the kinase domain of EGFR (codons 714 and 794) were identified. These genetic alterations were not previously described as mechanism of de-novo or acquired resistance. Consequently, further studies are needed to understand whether these mutations can confer resistance to antiEGFR therapy. 
Altogether these results demonstrate that heterogeneity is a feature of resistance to anti-EGFR therapy in CRC and that intra-tumor molecular complexity is even more evident in the contest of acquired resistance (Fig. 3 and Table 1). We postulate that the effect of pharmacological treatment represents a selective pressure, which allows the selection of (pre-existing) sub-clones that confer resistance to the drug. If this is the case, a number of questions arise. Is the presence of the resistant alleles a completely stochastic process? Or does a tumor maintain a reservoir of these sub-clones? Furthermore, why were these mutations not selected before the drug pressure similarly to those that confer primary resistance? It is conceivable that sub-clones that emerge after drug treatment are less fit in the untreated tumor and acquire fitness as a consequence of adaptation to the perturbation induced by the treatment itself. This event has been previously shown to occur in other cancer types. Chmielicki and colleagues demonstrated that erlotinib resistant NSCLC cells grew slower than their sensitive counterparts and, interestingly, resistance was not maintained in the absence of drug (80). A similar phenomenon has been described for $B R A F$ mutant melanoma cells, which become resistant to vemurafenib through expression of EGFR (81).

These data also enlighten the importance of the use of high sensitivity sequencing technologies for the detection of mutant alleles in CRC samples. A considerable fraction of patients that is eligible for anti-EGFR treatment develop secondary resistance in a very short timeframe. This could be explained as a higher frequency of pre-existing resistant clones in the initial population, which cannot be detected by standard sequencing but could be found with more sensitive technologies.

The overall compendium of molecular mechanisms driving acquired resistance to cetuximab and panitumumab is likely incomplete. While the role of $R A S$ mutations and MET gene amplification in conferring acquired resistance to EGFR blockade has been confirmed by several studies both in preclinical models and in patients $(62,75-79)$, candidate gene analysis does not always explain the mechanism by which a CRC becomes resistant to anti-EGFR therapy. Accordingly, further studies will likely characterize additional oncogenic alterations involved in acquired resistance to cetuximab and panitumumab in CRCs. Importantly, results from both cell models and clinical specimens indicate that every patient and possibly every metastatic lesion will develop several independent mechanisms of resistance to EGFR blockade $(38,76,78)$. It is therefore unlikely that we could obtain a complete profile of the molecular changes occurring in each metastatic patient who become resistant.

\section{Primary and acquired resistance to EGFR blockade: what is the difference?}

\section{The primary $=$ secondary rule}

EGFR targeted therapies are commonly used in the treatment of different tumor types of epithelial origin including NSCLC and CRC (82). Although the role of EGFR in the pathogenesis of these two 
cancer is distinct (in NCSLC EGFR is activated by mutations, while in CRC it is stimulated by ligands), interesting observations can be made comparing these two malignancies.

The mechanisms of acquired resistance to anti-EGFR antibodies in CRC can be broadly categorized in three groups (Fig 4). The first includes mutations that disrupt binding of cetuximab (or panitumumab) to the EGFR. This mechanism is analogous to the T790M mutations that emergence when NSCLC are treated with the kinase inhibitors erlotinib and gefitinib and render the receptor insensitive to the drug (83). The second mechanism involves pathway bypass mutations, such as KRAS or BRAF alterations. These are most common in CRC treated with anti EGFR antibodies but have been occasionally found also in NSCLC treated with EGFR TKi (84). The third mechanism is common to both tumors and involves activation of parallel pathways driven by RTK such as MET or ERBB2 $(85,86)$.

Nearly all the genetic alterations, which sustain de-novo resistance to EGFR blockade in mCRC, have also been identified as mechanisms of acquired resistance. Vice versa, mechanisms defined in the secondary setting can be also validated as primary resistance mechanisms. What are the implications of these findings? The striking overlap of primary and acquired resistance to EGFR blockade likely indicates that the selection applied by anti EGFR monoclonal antibodies to CRC cells is possibly analogous to the selective pressure exerted by the environment during cancer progression. It is conceivable that the pressure that selects for KRAS, NRAS or BRAF mutations must act in a similar manner in both settings. What are these pressures? We speculate that during the transition from adenoma to carcinoma (that is when RAS mutation events are thought to occur in the colorectal tumorigenesis sequence) a sudden lack of EGFR activation triggers the outgrowth of clones that are EGFR independent, but still dependent on its downstream signaling. Indeed, it is known that intestinal epithelial cells depend upon EGFR ligands (87). We speculate that a sudden loss in availability of EGFR ligands during the adenoma-carcinoma sequence selects for cancerous cells carrying RAS mutations. In a few instances, CRC cells overcome this pressure not by acquiring downstream pathway mutations but by gaining the ability to self-produce the EGFR ligands needed to sustain pathway activation. Such tumors maintain dependency/sensitivity to EGFR blockade in the later stages of CRC progression and define the subset of patients that obtain a clinical benefit from cetuximab and panitumumab.

\section{The primary = secondary rule has exceptions}

The EGFR extracellular domain mutation S492R represents the most notable exception to the primary = secondary rule. The S492R allele has never been detected to date in untreated CRC (88) and is apparently found only in CRC samples from patients who have been previously exposed to cetuximab. This is consistent with the hypothesis that this allele evolves as cells strive to evade the EGFR blockade imposed by the monoclonal antibody cetuximab, and accordingly remain sensitive 
to panitumumab which binds to a different EGFR epitope located on the extracellular domain of EGFR.

Even more intriguing is the other exception to primary=acquired rule. Remarkably, the relative frequency of individual KRAS alleles is similar but not identical in the primary and acquired resistance. For instance, mutations of codon 61 in either the KRAS or NRAS genes are more prevalent in the acquired than in the primary resistance setting (79). This suggests that the selective pressure which result in the acquisition of KRAS mutations during the transition from adenoma to carcinoma is again similar but not identical to the one applied by EGFR blockade (Fig. 4).

\section{Genetic heterogeneity and biochemical convergence}

\section{All roads lead to Rome}

In colorectal tumors that respond and then relapse after anti-EGFR treatment several genetic alterations concomitantly emerge. This phenomenon is best observed by analyzing circulating free DNA from patients at relapse (62,76-79), which offer a wide-angle perspective of the overall heterogeneity of the disease. This indicates that drug treatment triggers the evolution of multiple sub-clones each carrying distinct genetic alterations. Not unlike classical Darwinian evolution, the concomitant presence of several escape mechanisms reflects the high level of molecular heterogeneity present in each metastatic site, which enables the evolutionary processes.

The evolution of secondary resistance to anti-EGFR therapy can be defined as the consequence of a perturbation in a system, in which the initial equilibrium is based on cells, which are highly dependent on EGFR signaling. The finding that most of the mutations which emergence upon treatment involve genes which are direct members of the EGFR pathway (EGFR, KRAS, NRAS or BRAF) indicates that to escape the perturbation the cells must settle on a new balance which is (has to be) again based on a certain level of EGFR signaling output.

This hypothesis is supported by biochemical analysis of cell models of CRC that developed resistance to EGFR blockade regardless of the gene/mutation that confers resistance; the net output was always sustained activation of MEK and ERK, thus defining an example of convergent evolution (78).

Convergent evolution occurs when different species phylogenetically unrelated but placed in the same kind of environment or stimuli, develop parallel morphological features. A classic example of convergent evolution is observed when unrelated species such as mammals, reptiles and birds evolved "mechanical" features (wings) to be able to fly. Analogously, we postulate that when EGFR 
blockade occurs in a CRC patient with multiple metastatic lesions, the drug pressure triggers the convergent biochemical evolution of independent clones each of which reactivate the EGFR signaling output. Accordingly, although individual metastases develop what appear to be genetically heterogeneous resistance mechanisms these are in-fact highly related as they are aimed at reactivating at the biochemical level the EGFR signaling pathway. These findings have several implications that are discussed in the next paragraph.

\section{Conclusions}

\section{Exploiting the knowledge: The preemptive strike hypothesis}

The awareness that solid tumors, which initially respond and then relapse to a targeted therapy, will eventually become highly molecularly heterogeneous poses a formidable therapeutic challenge. At first glance, it would seem arduous to overcome the multiple resistance mutations that arise in each individual patient. While the overall picture is looming and complex, this knowledge offers several opportunities, which may be therapeutically exploited. For example, in CRC patients who receive anti EGFR therapies the plethora of alterations that emerge at relapse biochemically converge to activate the EGFR-RAS-MAPK pathway. This knowledge can be exploited in several ways. First, it suggests that at relapse the distinct resistance mechanisms can be intercepted by interfering downstream in the pathway where the signal outputs generated by the distinct genetic events converge: in this case at the MAPK-ERK level. A second and possibly even more relevant implication is that it may be more challenging for a colorectal tumor to escape EGFR blockade if the initial treatment is designed to concomitantly block the signaling nodes which we now know provide an escape (resistance) route. We hypothesize that blocking from the beginning (without offering the tumor the possibility to first escape the initial treatment) the most probable escape route the time required to develop resistance will be extended. In this regards, it will be important to assess -initially in preclinical models- whether the time it takes for CRC cells to develop resistance to EGFR blockade is extended significantly when the probable resistance pathway output (MEK reactivation) is concomitantly tackled. We postulate that if the latter scenario is confirmed this theory will provide unique opportunities for the design of innovative clinical trials, which will not await the inevitable development of resistant clones but rather will attempt at their pre-emptive suppression. 


\section{Acknowledgments}

We wish to thank Elizabeth Cook for her help on the graphical realization of the figures of this review and Sabrina Arena, Giovanni Germano, Luca Lazzari, Giulia Siravegna and Mariangela Russo for providing valuable feedback and for critically reading the manuscript.

\section{Grant Support:}

Work in the authors' laboratories is supported by the European Community's Seventh Framework Programme under grant agreement no. 259015 COLTHERES (A.B. and S.S.); Associazione Italiana per la Ricerca sul Cancro (AIRC) IG grant no. 12812 (A.B.); AIRC MFAG no. 11349 (F.D.N.); "Farmacogenomica"-5 per mille 2009 MIUR-Fondazione Piemontese per la Ricerca sul Cancro-ONLUS (F.D.N.); AIRC 2010 Special Program Molecular Clinical Oncology 5 per mille, project no. 9970 (A.B. and S.S.); Ministero dell'Istruzione, dell'Università e della Ricerca, progetto PRIN (A.B.); Progetti di Ateneo-2011, Università di Torino (ORTO11RKTW to A.B.); grant from Oncologia Ca' Granda (OCGO) Fondazione (S.S.); grant "Identification and monitoring of gene mutations in peripheral blood and urine as a diagnostic tool for patients with solid tumors from Regione Lombardia and Ministero Salute (S.S.). Grant "Liquid Biopsy"-5 per mille 2010 Ministero della Salute -Fondazione Piemontese per la Ricerca sul Cancro-ONLUS (A.B.); and grant "Precision Oncology"-5 per mille 2011 Ministero della Salute -Fondazione Piemontese per la Ricerca sul Cancro-ONLUS (A.B.). 


\section{References}

1. Nowell PC. The clonal evolution of tumor cell populations. Science 1976;194(4260):23-8.

2. Gerlinger M, Rowan AJ, Horswell S, Larkin J, Endesfelder D, Gronroos E, et al. Intratumor heterogeneity and branched evolution revealed by multiregion sequencing. N Engl J Med 2012;366(10):883-92.

3. Kreso A, O'Brien CA, van Galen P, Gan OI, Notta F, Brown AM, et al. Variable clonal repopulation dynamics influence chemotherapy response in colorectal cancer. Science 2013;339(6119):543-8.

4. Siegel R, Desantis C, Jemal A. Colorectal cancer statistics, 2014. CA: a cancer journal for clinicians 2014;64(2):104-17.

5. Vermaat JS, Nijman IJ, Koudijs MJ, Gerritse FL, Scherer SJ, Mokry M, et al. Primary colorectal cancers and their subsequent hepatic metastases are genetically different: implications for selection of patients for targeted treatment. Clin Cancer Res 2012;18(3):688-99.

6. Lee SY, Haq F, Kim D, Jun C, Jo HJ, Ahn SM, et al. Comparative genomic analysis of primary and synchronous metastatic colorectal cancers. PLOS ONE 2014;9(3):e90459.

7. Saltz LB, Meropol NJ, Loehrer PJ, Sr., Needle MN, Kopit J, Mayer RJ. Phase II trial of cetuximab in patients with refractory colorectal cancer that expresses the epidermal growth factor receptor. J Clin Oncol 2004;22(7):1201-8.

8. Van Cutsem E, Peeters M, Siena S, Humblet Y, Hendlisz A, Neyns B, et al. Open-label phase III trial of panitumumab plus best supportive care compared with best supportive care alone in patients with chemotherapy-refractory metastatic colorectal cancer. J Clin Oncol 2007;25(13):1658-64.

9. Moroni M, Veronese S, Benvenuti S, Marrapese G, Sartore-Bianchi A, Di Nicolantonio F, et al. Gene copy number for epidermal growth factor receptor (EGFR) and clinical response to antiEGFR treatment in colorectal cancer: a cohort study. The lancet oncology 2005;6(5):279-86.

10. Lievre A, Bachet JB, Le Corre D, Boige V, Landi B, Emile JF, et al. KRAS mutation status is predictive of response to cetuximab therapy in colorectal cancer. Cancer Res 2006;66(8):3992-5.

11. Benvenuti S, Sartore-Bianchi A, Di Nicolantonio F, Zanon C, Moroni M, Veronese S, et al. Oncogenic activation of the RAS/RAF signaling pathway impairs the response of metastatic colorectal cancers to anti-epidermal growth factor receptor antibody therapies. Cancer Res 2007;67(6):2643-8.

12. Amado RG, Wolf M, Peeters M, Van Cutsem E, Siena S, Freeman DJ, et al. Wild-type KRAS is required for panitumumab efficacy in patients with metastatic colorectal cancer. J Clin Oncol 2008;26(10):1626-34.

13. Karapetis CS, Khambata-Ford S, Jonker DJ, O'Callaghan CJ, Tu D, Tebbutt NC, et al. K-ras mutations and benefit from cetuximab in advanced colorectal cancer. N Engl J Med 2008;359(17):1757-65.

14. Bokemeyer C, Bondarenko I, Makhson A, Hartmann JT, Aparicio J, de Braud F, et al. Fluorouracil, leucovorin, and oxaliplatin with and without cetuximab in the first-line treatment of metastatic colorectal cancer. J Clin Oncol 2009;27(5):663-71.

15. Van Cutsem E, Kohne CH, Hitre E, Zaluski J, Chang Chien CR, Makhson A, et al. Cetuximab and chemotherapy as initial treatment for metastatic colorectal cancer. N Engl J Med 2009;360(14):1408-17.

16. Edkins S, O'Meara S, Parker A, Stevens C, Reis M, Jones S, et al. Recurrent KRAS codon 146 mutations in human colorectal cancer. Cancer Biol Ther 2006;5(8):928-32.

17. Janakiraman M, Vakiani E, Zeng Z, Pratilas CA, Taylor BS, Chitale D, et al. Genomic and biological characterization of exon 4 KRAS mutations in human cancer. Cancer Res 2010;70(14):5901-11.

18. Smith G, Bounds R, Wolf H, Steele RJ, Carey FA, Wolf CR. Activating K-Ras mutations outwith 'hotspot' codons in sporadic colorectal tumours - implications for personalised cancer medicine. $\mathrm{Br}$ J Cancer 2010;102(4):693-703.

19. Vaughn CP, Zobell SD, Furtado LV, Baker CL, Samowitz WS. Frequency of KRAS, BRAF, and NRAS mutations in colorectal cancer. Genes Chromosomes Cancer 2011;50(5):307-12.

20. De Roock W, Claes B, Bernasconi D, De Schutter J, Biesmans B, Fountzilas G, et al. Effects of KRAS, BRAF, NRAS, and PIK3CA mutations on the efficacy of cetuximab plus chemotherapy in 
chemotherapy-refractory metastatic colorectal cancer: a retrospective consortium analysis. Lancet Oncol 2010;11(8):753-62.

21. Loupakis F, Ruzzo A, Cremolini C, Vincenzi B, Salvatore L, Santini D, et al. KRAS codon 61, 146 and BRAF mutations predict resistance to cetuximab plus irinotecan in KRAS codon 12 and 13 wild-type metastatic colorectal cancer. Br J Cancer 2009;101(4):715-21.

22. Molinari F, Felicioni L, Buscarino M, De Dosso S, Buttitta F, Malatesta S, et al. Increased detection sensitivity for KRAS mutations enhances the prediction of anti-EGFR monoclonal antibody resistance in metastatic colorectal cancer. Clinical cancer research : an official journal of the American Association for Cancer Research 2011;17(14):4901-14.

23. Peeters M, Oliner KS, Parker A, Siena S, Van Cutsem E, Huang J, et al. Massively parallel tumor multigene sequencing to evaluate response to panitumumab in a randomized phase III study of metastatic colorectal cancer. Clin Cancer Res 2013;19(7):1902-12.

24. Douillard JY, Oliner KS, Siena S, Tabernero J, Burkes R, Barugel M, et al. Panitumumab-FOLFOX4 treatment and RAS mutations in colorectal cancer. N Engl J Med 2013;369(11):1023-34.

25. Schwartzberg LS, Rivera F, Karthaus M, Fasola G, Canon JL, Hecht JR, et al. PEAK: A Randomized, Multicenter Phase II Study of Panitumumab Plus Modified Fluorouracil, Leucovorin, and Oxaliplatin (mFOLFOX6) or Bevacizumab Plus mFOLFOX6 in Patients With Previously Untreated, Unresectable, Wild-Type KRAS Exon 2 Metastatic Colorectal Cancer. J Clin Oncol 2014.

26. Stintzing S, Jung A, Rossius L, Modest DP, Fischer von Weikersthal L, Decker T, et al. Analysis of KRAS/NRAS and BRAF mutations in FIRE-3: A randomized phase III study of FOLFIRI plus cetuximab or bevacizumab as first-line treatment for wild-type (WT) KRAS (exon 2) metastatic colorectal cancer (mCRC) patients. . Eur J Cancer. Volume 49 (Suppl. 3). European Cancer Congress 2013; Amsterdam, The Netherlands.2013. p Abstract LBA 17.

27. Di Nicolantonio F, Martini M, Molinari F, Sartore-Bianchi A, Arena S, Saletti P, et al. Wild-type BRAF is required for response to panitumumab or cetuximab in metastatic colorectal cancer. $J$ Clin Oncol 2008;26(35):5705-12.

28. Jhawer M, Goel S, Wilson AJ, Montagna C, Ling YH, Byun DS, et al. PIK3CA mutation/PTEN expression status predicts response of colon cancer cells to the epidermal growth factor receptor inhibitor cetuximab. Cancer Res 2008;68(6):1953-61.

29. Bertotti A, Migliardi G, Galimi F, Sassi F, Torti D, Isella C, et al. A molecularly annotated platform of patient-derived xenografts ("xenopatients") identifies HER2 as an effective therapeutic target in cetuximab-resistant colorectal cancer. Cancer Discov 2011;1(6):508-23.

30. Laurent-Puig P, Cayre A, Manceau G, Buc E, Bachet JB, Lecomte T, et al. Analysis of PTEN, BRAF, and EGFR status in determining benefit from cetuximab therapy in wild-type KRAS metastatic colon cancer. J Clin Oncol 2009;27(35):5924-30.

31. Souglakos J, Philips J, Wang R, Marwah S, Silver M, Tzardi M, et al. Prognostic and predictive value of common mutations for treatment response and survival in patients with metastatic colorectal cancer. Br J Cancer 2009;101(3):465-72.

32. Valtorta E, Misale S, Sartore-Bianchi A, Nagtegaal ID, Paraf F, Lauricella C, et al. KRAS gene amplification in colorectal cancer and impact on response to EGFR-targeted therapy. Int J Cancer 2013;133(5):1259-65.

33. Mekenkamp LJ, Tol J, Dijkstra JR, de Krijger I, Vink-Borger ME, van Vliet S, et al. Beyond KRAS mutation status: influence of KRAS copy number status and microRNAs on clinical outcome to cetuximab in metastatic colorectal cancer patients. BMC Cancer 2012;12:292.

34. Comprehensive molecular characterization of human colon and rectal cancer. Nature 2012;487(7407):330-7.

35. Yonesaka K, Zejnullahu K, Okamoto I, Satoh T, Cappuzzo F, Souglakos J, et al. Activation of ERBB2 signaling causes resistance to the EGFR-directed therapeutic antibody cetuximab. Sci Transl Med 2011;3(99):99ra86.

36. Cappuzzo F, Varella-Garcia M, Finocchiaro G, Skokan M, Gajapathy S, Carnaghi C, et al. Primary resistance to cetuximab therapy in EGFR FISH-positive colorectal cancer patients. Br J Cancer 2008;99(1):83-9. 
37. Inno A, Di Salvatore M, Cenci T, Martini M, Orlandi A, Strippoli A, et al. Is there a role for IGF1R and c-MET pathways in resistance to cetuximab in metastatic colorectal cancer? Clinical colorectal cancer 2011;10(4):325-32.

38. Bardelli A, Corso S, Bertotti A, Hobor S, Valtorta E, Siravegna G, et al. Amplification of the MET receptor drives resistance to anti-EGFR therapies in colorectal cancer. Cancer Discov 2013;3(6):65873.

39. Iwamoto S, Hazama S, Kato T, Miyake Y, Fukunaga M, Matsuda C, et al. Multicenter Phase II Study of Second-line Cetuximab plus Folinic Acid/5-Fluorouracil/Irinotecan (FOLFIRI) in KRAS Wild-type Metastatic Colorectal Cancer: The FLIER Study. Anticancer research 2014;34(4):1967-73.

40. Karapetis CS, Jonker D, Daneshmand M, Hanson JE, O'Callaghan CJ, Marginean C, et al. PIK3CA, $B R A F$, and PTEN status and benefit from cetuximab in the treatment of advanced colorectal cancer-results from NCIC CTG/AGITG CO.17. Clin Cancer Res 2014;20(3):744-53.

41. Perkins G, Lievre A, Ramacci C, Meatchi T, de Reynies A, Emile JF, et al. Additional value of EGFR downstream signaling phosphoprotein expression to KRAS status for response to anti-EGFR antibodies in colorectal cancer. Int J Cancer 2010;127(6):1321-31.

42. Perrone F, Lampis A, Orsenigo M, Di Bartolomeo M, Gevorgyan A, Losa M, et al. PI3KCA/PTEN deregulation contributes to impaired responses to cetuximab in metastatic colorectal cancer patients. Ann Oncol 2009;20(1):84-90.

43. Prenen H, De Schutter J, Jacobs B, De Roock W, Biesmans B, Claes B, et al. PIK3CA mutations are not a major determinant of resistance to the epidermal growth factor receptor inhibitor cetuximab in metastatic colorectal cancer. Clin Cancer Res 2009;15(9):3184-8.

44. Sartore-Bianchi A, Di Nicolantonio F, Nichelatti M, Molinari F, De Dosso S, Saletti P, et al. Multideterminants analysis of molecular alterations for predicting clinical benefit to EGFR-targeted monoclonal antibodies in colorectal cancer. PLoS ONE 2009;4(10):e7287.

45. Smith CG, Fisher D, Claes B, Maughan TS, Idziaszczyk S, Peuteman G, et al. Somatic profiling of the epidermal growth factor receptor pathway in tumors from patients with advanced colorectal cancer treated with chemotherapy +/- cetuximab. Clin Cancer Res 2013;19(15):4104-13.

46. Soeda H, Shimodaira H, Watanabe M, Suzuki T, Gamoh M, Mori T, et al. Clinical usefulness of KRAS, BRAF, and PIK3CA mutations as predictive markers of cetuximab efficacy in irinotecan- and oxaliplatin-refractory Japanese patients with metastatic colorectal cancer. Int J Clin Oncol 2013;18(4):670-7.

47. Spindler KL, Pallisgaard N, Lindebjerg J, Frifeldt SK, Jakobsen A. EGFR related mutational status and association to clinical outcome of third-line cetuximab-irinotecan in metastatic colorectal cancer. BMC Cancer 2011;11:107.

48. Pentheroudakis G, Kotoula V, De Roock W, Kouvatseas G, Papakostas P, Makatsoris T, et al. Biomarkers of benefit from cetuximab-based therapy in metastatic colorectal cancer: interaction of EGFR ligand expression with RAS/RAF, PIK3CA genotypes. BMC Cancer 2013;13:49.

49. Frattini $M$, Saletti $P$, Romagnani E, Martin V, Molinari F, Ghisletta $M$, et al. PTEN loss of expression predicts cetuximab efficacy in metastatic colorectal cancer patients. Br J Cancer 2007;97(8):113945.

50. Loupakis F, Pollina L, Stasi I, Ruzzo A, Scartozzi M, Santini D, et al. PTEN expression and KRAS mutations on primary tumors and metastases in the prediction of benefit from cetuximab plus irinotecan for patients with metastatic colorectal cancer. J Clin Oncol 2009;27(16):2622-9.

51. Molinari F, Martin V, Saletti P, De Dosso S, Spitale A, Camponovo A, et al. Differing deregulation of EGFR and downstream proteins in primary colorectal cancer and related metastatic sites may be clinically relevant. Br J Cancer 2009.

52. Saridaki Z, Tzardi M, Papadaki C, Sfakianaki M, Pega F, Kalikaki A, et al. Impact of KRAS, BRAF, PIK3CA mutations, PTEN, AREG, EREG expression and skin rash in $>/=2$ line cetuximab-based therapy of colorectal cancer patients. PLoS ONE 2011;6(1):e15980.

53. Sartore-Bianchi A, Martini M, Molinari F, Veronese S, Nichelatti M, Artale S, et al. PIK3CA mutations in colorectal cancer are associated with clinical resistance to EGFR-targeted monoclonal antibodies. Cancer Res 2009;69(5):1851-7. 
54. Sood A, McClain D, Maitra R, Basu-Mallick A, Seetharam R, Kaubisch A, et al. PTEN gene expression and mutations in the PIK3CA gene as predictors of clinical benefit to anti-epidermal growth factor receptor antibody therapy in patients with KRAS wild-type metastatic colorectal cancer. Clinical colorectal cancer 2012;11(2):143-50.

55. Tol J, Dijkstra JR, Klomp M, Teerenstra S, Dommerholt M, Vink-Borger ME, et al. Markers for EGFR pathway activation as predictor of outcome in metastatic colorectal cancer patients treated with or without cetuximab. Eur J Cancer 2010;46(11):1997-2009.

56. Negri FV, Bozzetti C, Lagrasta CA, Crafa P, Bonasoni MP, Camisa R, et al. PTEN status in advanced colorectal cancer treated with cetuximab. Br J Cancer 2010;102(1):162-4.

57. Kosmidou V, Oikonomou E, Vlassi M, Avlonitis S, Katseli A, Tsipras I, et al. Tumor heterogeneity revealed by KRAS, BRAF, and PIK3CA pyrosequencing: KRAS and PIK3CA intratumor mutation profile differences and their therapeutic implications. Hum Mutat 2014;35(3):329-40.

58. Richman SD, Chambers P, Seymour MT, Daly C, Grant S, Hemmings G, et al. Intra-tumoral heterogeneity of KRAS and BRAF mutation status in patients with advanced colorectal cancer (aCRC) and cost-effectiveness of multiple sample testing. Anal Cell Pathol (Amst) 2011;34(1-2):61-6.

59. Tsiatis AC, Norris-Kirby A, Rich RG, Hafez MJ, Gocke CD, Eshleman JR, et al. Comparison of Sanger sequencing, pyrosequencing, and melting curve analysis for the detection of KRAS mutations: diagnostic and clinical implications. J Mol Diagn 2010;12(4):425-32.

60. Tougeron D, Lecomte T, Pages JC, Villalva C, Collin C, Ferru A, et al. Effect of low-frequency KRAS mutations on the response to anti-EGFR therapy in metastatic colorectal cancer. Ann Oncol 2013;24(5):1267-73.

61. Bando H, Yoshino T, Tsuchihara K, Ogasawara N, Fuse N, Kojima T, et al. KRAS mutations detected by the amplification refractory mutation system-Scorpion assays strongly correlate with therapeutic effect of cetuximab. Br J Cancer 2011;105(3):403-6.

62. Misale S, Yaeger R, Hobor S, Scala E, Janakiraman M, Liska D, et al. Emergence of KRAS mutations and acquired resistance to anti-EGFR therapy in colorectal cancer. Nature 2012;486(7404):532-6.

63. Hobor S, Van Emburgh BO, Crowley E, Misale S, Di Nicolantonio F, Bardelli A. TGF- $\alpha$ and amphiregulin paracrine network promotes resistance to EGFR blockade in colorectal cancer cells. Clin Cancer Res 2014.

64. Sartore-Bianchi A, Moroni M, Veronese S, Carnaghi C, Bajetta E, Luppi G, et al. Epidermal growth factor receptor gene copy number and clinical outcome of metastatic colorectal cancer treated with panitumumab. J Clin Oncol 2007;25(22):3238-45.

65. Bertotti A, Migliardi G, Galimi F, Sassi F, Torti D, Isella C, et al. A molecularly annotated platform of patient-derived xenografts ("xenopatients") identifies HER2 as an effective therapeutic target in cetuximab-resistant colorectal cancer. Cancer Discov 2011;1(6):508-23.

66. Laurent-Puig P, Cayre A, Manceau G, Buc E, Bachet JB, Lecomte T, et al. Analysis of PTEN, BRAF, and EGFR status in determining benefit from cetuximab therapy in wild-type KRAS metastatic colon cancer. J Clin Oncol 2009;27(35):5924-30.

67. Scartozzi M, Bearzi I, Mandolesi A, Pierantoni C, Loupakis F, Zaniboni A, et al. Epidermal Growth Factor Receptor (EGFR) gene copy number (GCN) correlates with clinical activity of irinotecancetuximab in K-RAS wild-type colorectal cancer: a fluorescence in situ (FISH) and chromogenic in situ hybridization (CISH) analysis. BMC Cancer 2009;9:303.

68. Personeni N, Fieuws S, Piessevaux H, De Hertogh G, De Schutter J, Biesmans B, et al. Clinical usefulness of EGFR gene copy number as a predictive marker in colorectal cancer patients treated with cetuximab: a fluorescent in situ hybridization study. Clin Cancer Res 2008;14(18):5869-76.

69. Cappuzzo F, Finocchiaro G, Rossi E, Jänne PA, Carnaghi C, Calandri C, et al. EGFR FISH assay predicts for response to cetuximab in chemotherapy refractory colorectal cancer patients. Ann Oncol 2008;19(4):717-23.

70. Sartore-Bianchi A, Fieuws S, Veronese S, Moroni M, Personeni N, Frattini M, et al. Standardisation of EGFR FISH in colorectal cancer: results of an international interlaboratory reproducibility ring study. J Clin Pathol 2012;65(3):218-23. 
71. Fan Z, Masui H, Altas I, Mendelsohn J. Blockade of epidermal growth factor receptor function by bivalent and monovalent fragments of 225 anti-epidermal growth factor receptor monoclonal antibodies. Cancer Res 1993;53(18):4322-8.

72. Fan Z, Lu Y, Wu X, Mendelsohn J. Antibody-induced epidermal growth factor receptor dimerization mediates inhibition of autocrine proliferation of A431 squamous carcinoma cells. The Journal of biological chemistry 1994;269(44):27595-602.

73. Montagut C, Dalmases A, Bellosillo B, Crespo M, Pairet S, Iglesias M, et al. Identification of a mutation in the extracellular domain of the Epidermal Growth Factor Receptor conferring cetuximab resistance in colorectal cancer. Nat Med 2012;18(2):221-3.

74. Voigt M, Braig F, Göthel M, Schulte A, Lamszus K, Bokemeyer C, et al. Functional dissection of the epidermal growth factor receptor epitopes targeted by panitumumab and cetuximab. Neoplasia 2012;14(11):1023-31.

75. Diaz LA, Jr., Sausen M, Fisher GA, Velculescu VE. Insights into therapeutic resistance from wholegenome analyses of circulating tumor DNA. Oncotarget 2013;4(10):1856-7.

76. Mohan S, Heitzer E, Ulz P, Lafer I, Lax S, Auer M, et al. Changes in colorectal carcinoma genomes under anti-EGFR therapy identified by whole-genome plasma DNA sequencing. PLoS Genet 2014;10(3):e1004271.

77. Diaz LA, Williams RT, Wu J, Kinde I, Hecht JR, Berlin J, et al. The molecular evolution of acquired resistance to targeted EGFR blockade in colorectal cancers. Nature 2012;486(7404):537-40.

78. Misale S, Arena S, Lamba S, Siravegna G, Lallo A, Hobor S, et al. Blockade of EGFR and MEK intercepts heterogeneous mechanisms of acquired resistance to anti-EGFR therapies in colorectal cancer. Sci Transl Med 2014;6(224):224ra26.

79. Bettegowda C, Sausen M, Leary RJ, Kinde I, Wang Y, Agrawal N, et al. Detection of circulating tumor DNA in early- and late-stage human malignancies. Sci TransI Med 2014;6(224):224ra24.

80. Chmielecki J, Foo J, Oxnard GR, Hutchinson K, Ohashi K, Somwar R, et al. Optimization of dosing for EGFR-mutant non-small cell lung cancer with evolutionary cancer modeling. Sci Transl Med 2011;3(90):90ra59.

81. Sun C, Wang L, Huang S, Heynen GJ, Prahallad A, Robert C, et al. Reversible and adaptive resistance to BRAF(V600E) inhibition in melanoma. Nature 2014;508(7494):118-22.

82. Ciardiello F, Tortora G. EGFR antagonists in cancer treatment. N Engl J Med 2008;358(11):1160-74.

83. Pao W, Miller VA, Politi KA, Riely GJ, Somwar R, Zakowski MF, et al. Acquired resistance of lung adenocarcinomas to gefitinib or erlotinib is associated with a second mutation in the EGFR kinase domain. PLoS Med 2005;2(3):e73.

84. Ohashi K, Sequist LV, Arcila ME, Moran T, Chmielecki J, Lin YL, et al. Lung cancers with acquired resistance to EGFR inhibitors occasionally harbor BRAF gene mutations but lack mutations in KRAS, NRAS, or MEK1. Proc Natl Acad Sci U S A 2012;109(31):E2127-33.

85. Engelman JA, Zejnullahu K, Mitsudomi T, Song Y, Hyland C, Park JO, et al. MET amplification leads to gefitinib resistance in lung cancer by activating ERBB3 signaling. Science 2007;316(5827):1039-43.

86. Yu HA, Arcila ME, Rekhtman N, Sima CS, Zakowski MF, Pao W, et al. Analysis of tumor specimens at the time of acquired resistance to EGFR-TKI therapy in 155 patients with EGFR-mutant lung cancers. Clin Cancer Res 2013;19(8):2240-7.

87. Harris RC, Chung E, Coffey RJ. EGF receptor ligands. Exp Cell Res 2003;284(1):2-13.

88. Esposito C, Rachiglio AM, La Porta ML, Sacco A, Roma C, lannaccone A, et al. The S492R EGFR ectodomain mutation is never detected in KRAS wild-type colorectal carcinoma before exposure to EGFR monoclonal antibodies. Cancer Biol Ther 2013;14(12):1143-6.

89. Patterson S, Peeters M, Siena S, Van Cutsem E, Humblet $Y$, Van Laethem J-L, et al. Comprehensive analysis of KRAS and NRAS mutations as predictive biomarkers for single agent panitumumab (pmab) response in a randomized, phase III metastatic colorectal cancer (mCRC) study (20020408). Volume 31. Journal Of Clinical Oncology: ; 2013.

90. Douillard JY, Siena S, Cassidy J, Tabernero J, Burkes R, Barugel M, et al. Randomized, phase III trial of panitumumab with infusional fluorouracil, leucovorin, and oxaliplatin (FOLFOX4) versus FOLFOX4 
alone as first-line treatment in patients with previously untreated metastatic colorectal cancer: the PRIME study. J Clin Oncol 2010;28(31):4697-705.

\section{Legend to Figures and Tables}

Figure 1: Development of the anti EGFR antibodies cetuximab and panitumumab in metastatic colorectal cancer.

The timeline charts the key steps of the anti-EGFR targeted therapies approval for metastatic colorectal cancer treatment together with the most significant discoveries that supported these achievements. FDA: US Food and Drug Administration; EMA: European Medicine Agency.

Figure 2: Prevalence of genetic alterations associated with de-novo resistance to anti-EGFR therapies in metastatic colorectal cancer

Donut-chart of the genetic alterations involved in primary resistance to EGFR targeted monoclonal antibodies in mCRC. KRAS wild type population represents the sum of the anti-EGFR therapies responders (around 10\%) and the fraction of patients who do not benefit from those treatments even in the absence of known primary resistance mutations. KRAS exons 3 and 4, NRAS exons 2 , 3, 4 and amplification of KRAS, HER2 and MET account for around $20 \%$ of mCRC patients which do not benefit from anti-EGFR treatment. Data were collected from: Vaughn et al. 2011 (19), Bertotti et al. 2011 (65), Valtorta et al. 2013 (32), Bardelli et al. 2013 (38), Study 20020408 (89), PRIME trial (24)(90), Schwartzberg et al. 2014 (25), FIRE-3 trial (26).

Figure 3: Molecular heterogeneity drives secondary resistance to anti-EGFR therapies in metastatic colorectal cancer

Response to anti-EGFR targeted therapies in metastatic colorectal cancer is accompanied by selection of pre-existing resistant clones present in the initial metastasis burden. Conceivably resistant clones can also emerge during treatment. Clones, carrying distinct molecular alterations such as KRAS, NRAS, EGFR and BRAF mutations or KRAS, HER2 or MET amplifications, can coexist in the same metastatic site or in different metastatic sites. CT scans were obtained from a CRC patient who showed the first response to cetuximab observed at Ospedale Niguarda Ca' Granda in 2001.

Figure 4: Molecular mechanisms of primary and secondary resistance to anti-EGFR therapies in metastatic colorectal cancer

The genetic mechanisms responsible for de-novo and acquired resistance largely overlap. With the exception of EGFR mutations, which were described only in the acquired setting, all of the genetic alterations defined as mechanism of de-novo resistance are also responsible for acquired resistance. Differences can be found in the frequency of individual genetic alterations, such as KRAS and NRAS exon 3 mutations, which occur more frequently in the acquired rather than in the de-novo setting.

Table 1: Summary of genetic alterations associated with secondary resistance to EGFR blockade in $\mathrm{mCRCs}$ 


\section{Figure 1}

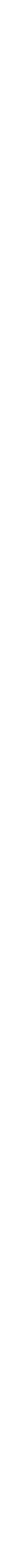

EMA restricts the use of cetuximab to patients with KRAS exon 2 wild-type 
Figure 2

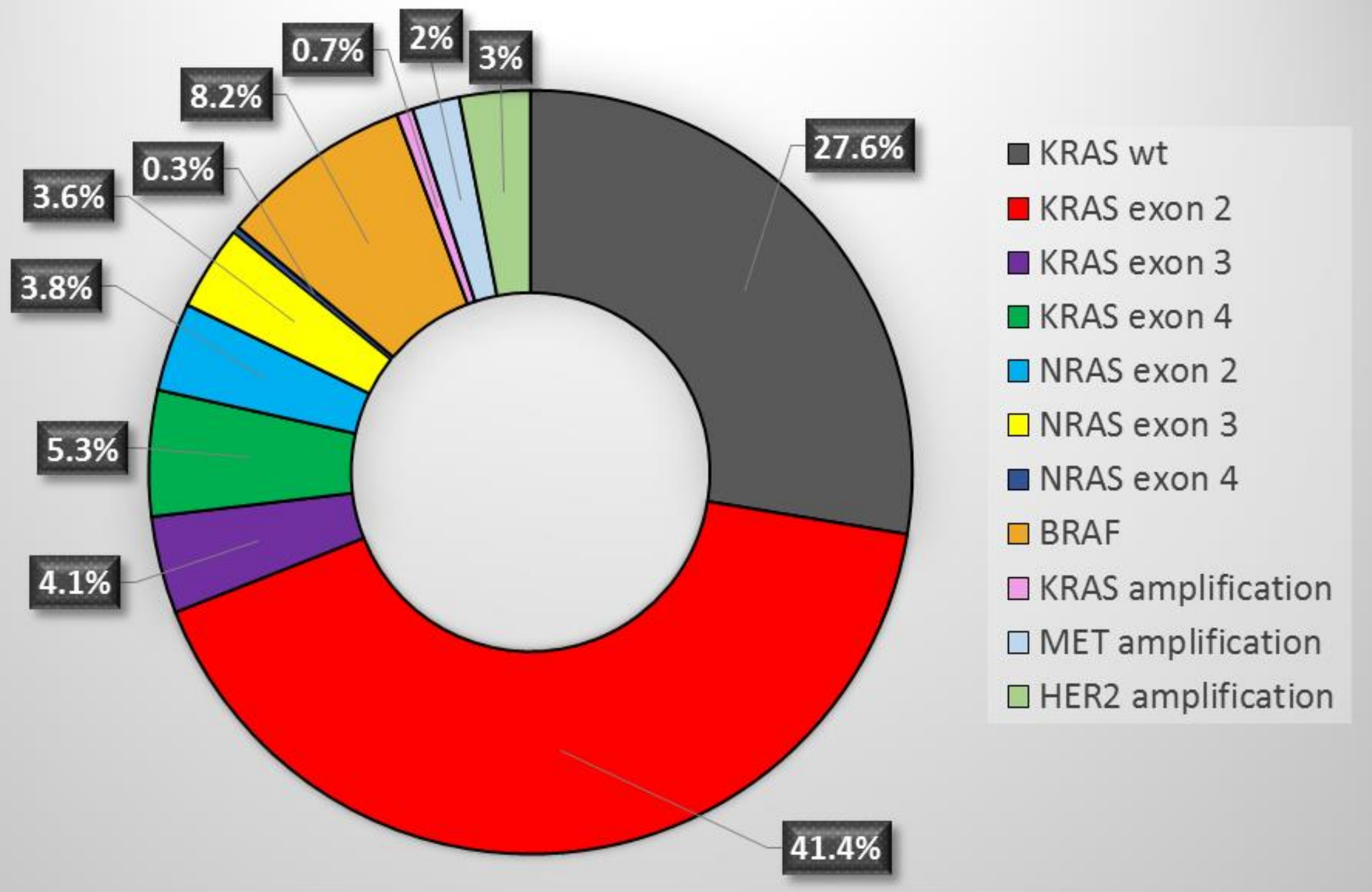




\section{Figure 3}

Molecular heterogeneity drives secondary resistance to anti EGFR therapies
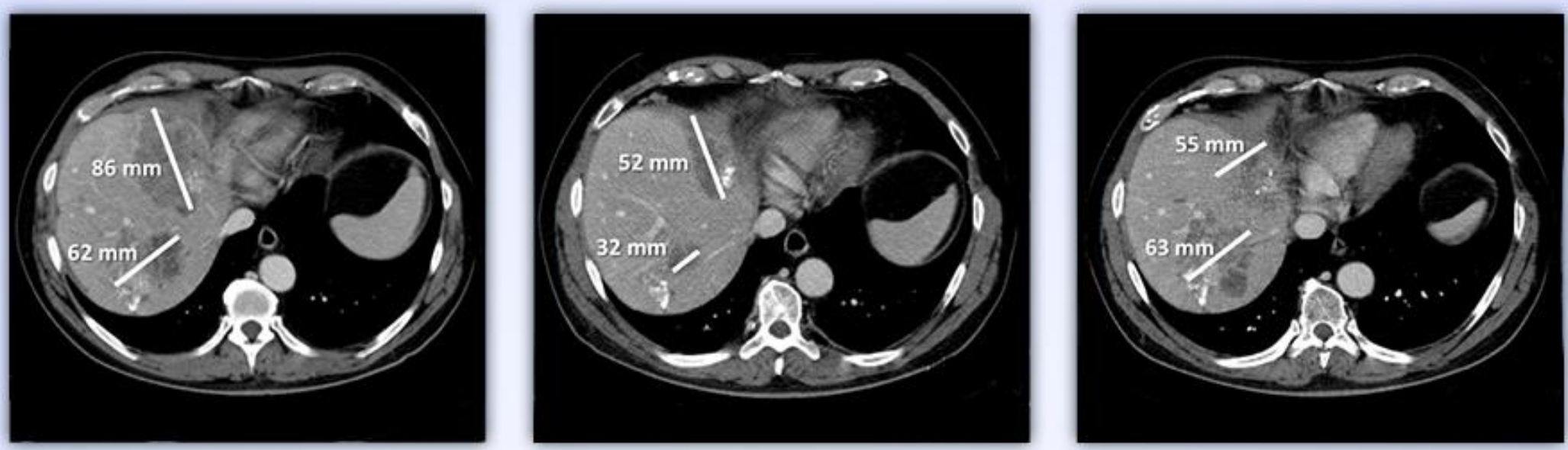

Baseline

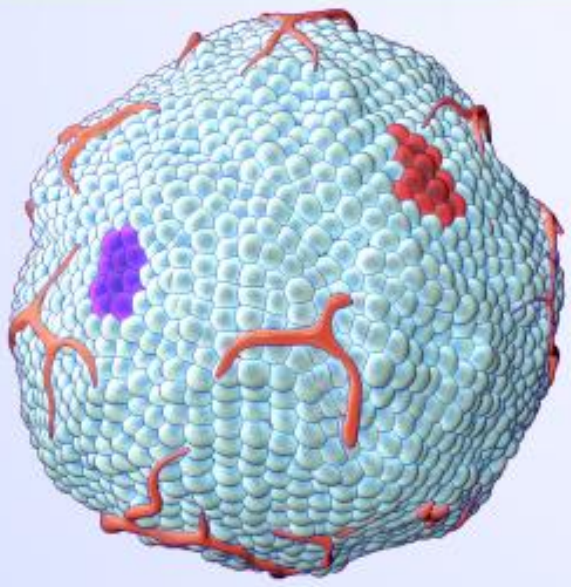

Partial response

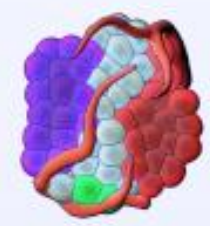

(

Sensitive tumor cell
000

Resistant tumor cells
Progression

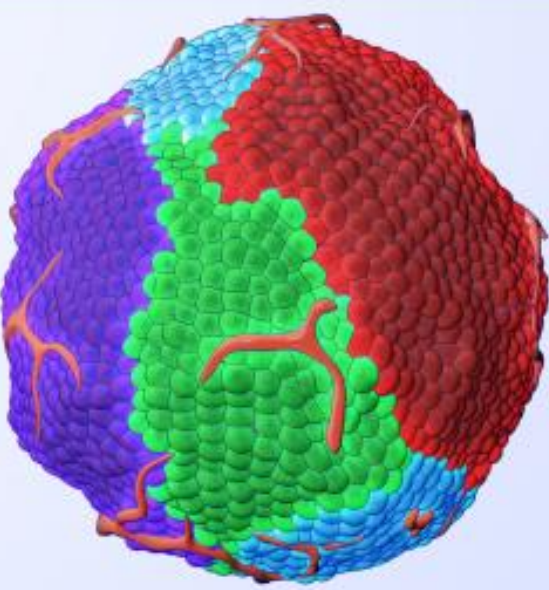


Figure 4

De-novo Resistance

MET, HER2, or KRAS amplification
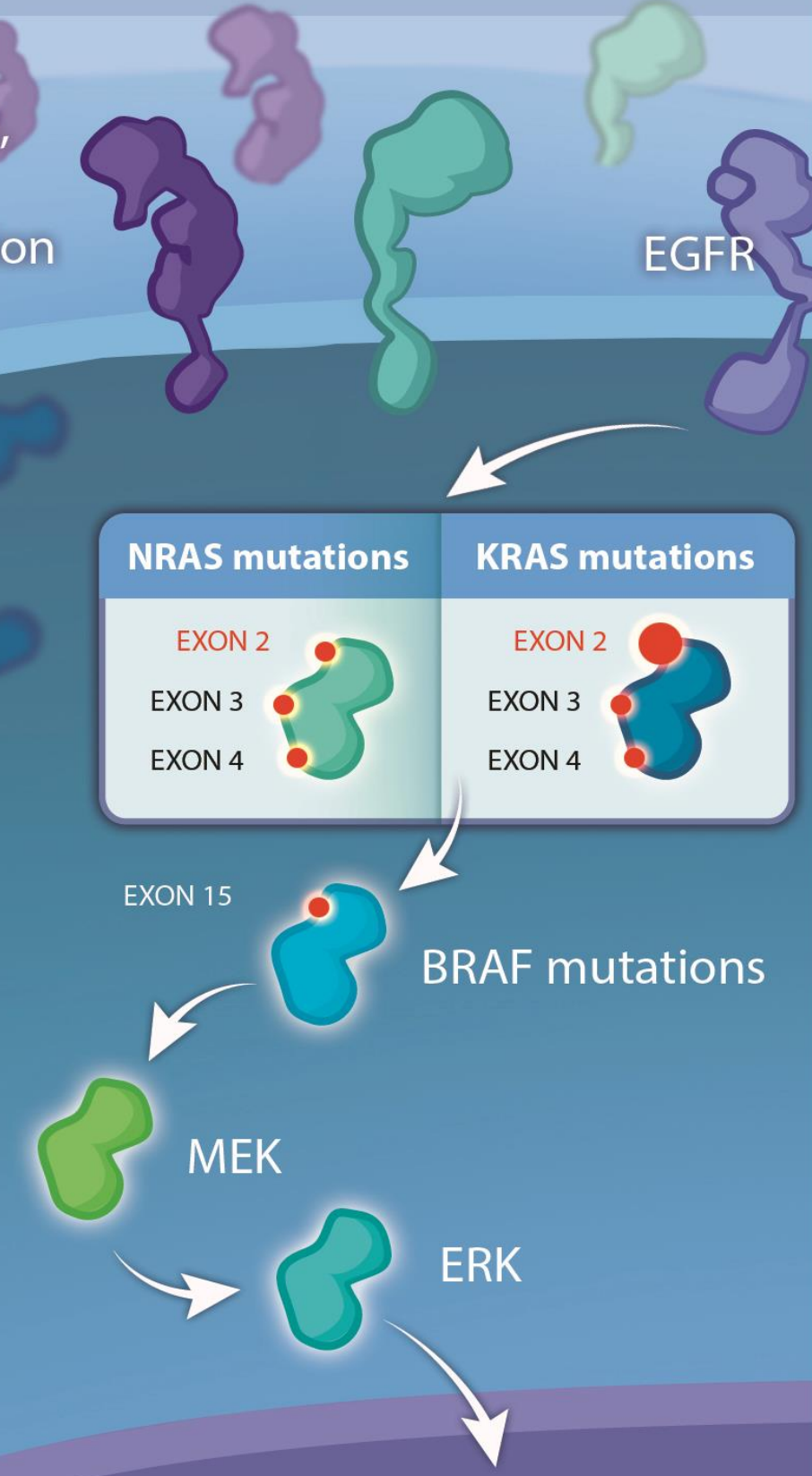

Acquired Resistance

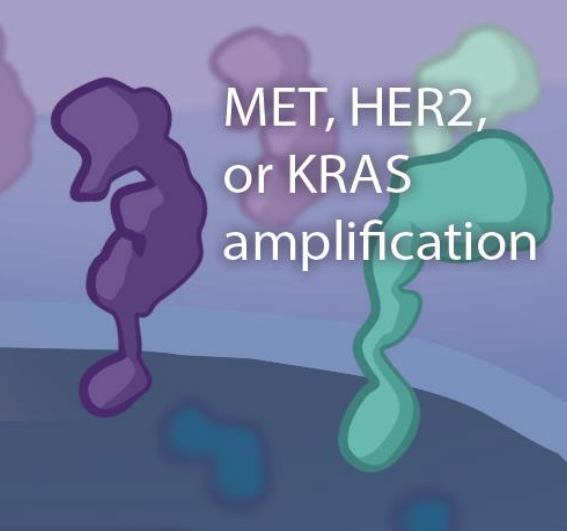

KRAS mutations NRAS mutations

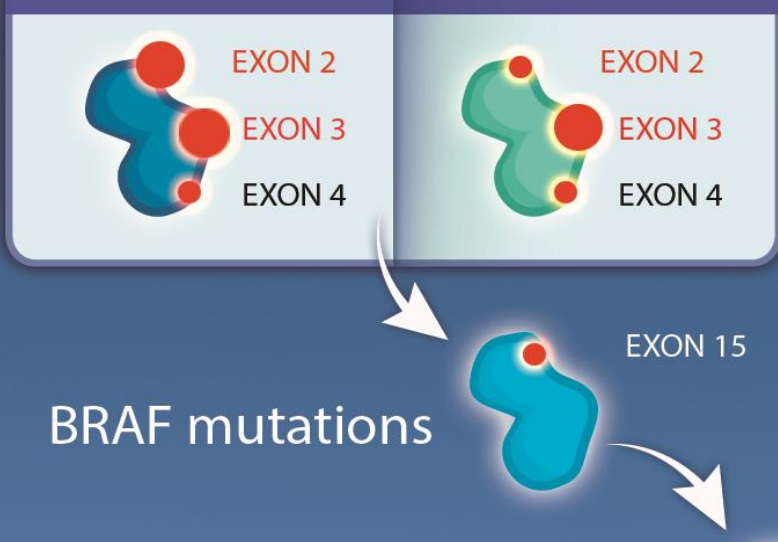

MEK

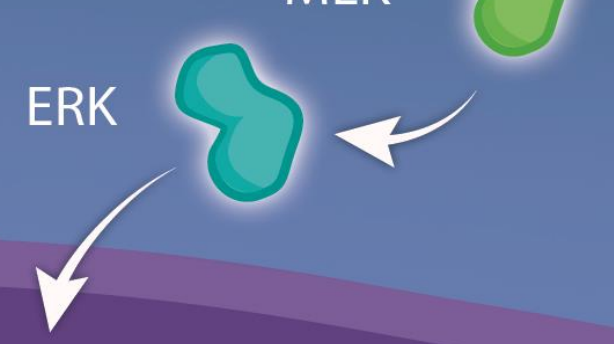




\section{Table 1}

\begin{tabular}{|c|c|c|c|c|}
\hline Reference/Study & $\begin{array}{c}\text { Genetic alterations at secondary } \\
\text { resistance }\end{array}$ & Tumor sample type & $\begin{array}{l}\text { Number of } \\
\text { patients }\end{array}$ & $\begin{array}{l}\text { Number of patients } \\
\text { displaying more than one } \\
\text { genetic alterations at } \\
\text { onset of resistance }\end{array}$ \\
\hline Yonesaka, 2011 & HER2 amplification & Tissue & $2 / 2$ & none \\
\hline \multirow{3}{*}{ Montagut, 2012} & EGFR mutations & Tissue & $2 / 10$ & \multirow{3}{*}{ none } \\
\hline & KRAS mutations & Tissue & $1 / 10$ & \\
\hline & BRAF mutations & Tissue & $1 / 10$ & \\
\hline Diaz, 2012 & KRAS mutations & Plasma & $9 / 24$ & $3 / 24$ \\
\hline \multirow{2}{*}{ Misale, 2012} & KRAS mutations & Plasma and Tissue & $5 / 11$ & \multirow{2}{*}{$1 / 11$} \\
\hline & KRAS amplification & Plasma and Tissue & $1 / 11$ & \\
\hline \multirow{2}{*}{ Bardelli, 2013} & MET amplification & Plasma and Tissue & $3 / 7$ & \multirow{2}{*}{ none } \\
\hline & KRAS mutations & Plasma and Tissue & $3 / 7$ & \\
\hline \multirow{4}{*}{ Bettegowda, 2014} & KRAS mutations & Plasma & $22 / 24$ & \multirow{4}{*}{$15 / 24$} \\
\hline & NRAS mutations & Plasma & $9 / 24$ & \\
\hline & BRAF mutations & Plasma & $1 / 24$ & \\
\hline & EGFR mutations & Plasma & $2 / 24$ & \\
\hline \multirow{2}{*}{ Misale, 2014} & KRAS mutations & Plasma & $3 / 4$ & \multirow{2}{*}{$3 / 4$} \\
\hline & NRAS mutations & Plasma & $2 / 4$ & \\
\hline \multirow{2}{*}{ Mohan, 2014} & KRAS amplification & Plasma & $4 / 10$ & \multirow{2}{*}{$1 / 10$} \\
\hline & MET amplification & Plasma & $1 / 10$ & \\
\hline
\end{tabular}

